\title{
THE NASA/INDUSTRY DESIGN ANALYSIS METHODS FOR VIBRATIONS (DAMVIBS) PROGRAM - MCDONNELL DOUGLAS HELICOPTER COMPANY ACHIEVEMENTS
}

\author{
Mostafa Toossi * \\ Richard Weisenburger ${ }^{\dagger}$ \\ Mostafa Hashemi-Kia \\ McDonnell Douglas Helicopter Company \\ Mesa, Arizona
}

\begin{abstract}
Abatract
This paper presents a summary of soine of the work performed by McDonnell Douglas Helicopter Company under NASA Langley-sponeored rotorcraft structural dynamica program known as DAMVIBS (Design Analysis Methode for VIBrationS). A set of guidelines which is applicable to dynamic modeling, analyois, teating, and correlation of both helicopter airframes and a large variety of structural finite element models is presented. Utilisation of these guidelines and the key features of their applications to vibration modeling of helicopter airfranies are discussed. Correlation otudiee with the teat data, together with the development and applications of set of efficient finite element model checkout procedures, are demonstrated on a large helicopter airframe finite element model. Fiually, the lessons learned and the benefits resulting from this program are oummarised.
\end{abstract}

\section{Background and Introduction}

Under a II. S. Government sponsored contracl, McDonnell Douglas Helicopter (MDHC) was one of the four helicopter manufacturers who participated in conducting $n$ atudy of finite element analysis of helicopter airframes for the enhancement of the technology base related to the area of airframe structural dynamic analysis. This work which was sponsored by the NASA Langley Research Center Structures Directorate, consisted of planning, development, and the documentation of modeling techniques and computational procedures. Additional tests were performed to obtain the necesary data to verify the finite element model and to develop procedures for correlation studies. The nodeling and shake test were performed on the McDonnell Douglas AH-64A (Apache) PV01 Helicopter. Initially, a atructural finite element model was prepared (1).

- Manager, Momber Ails.

t Reweareh Englineer, Mombor AHS.

Reoparch Endineer Momber AH8.
The guidelines were broken into three different categories which consisted of those used for modeling of the atiffness characteristics, representation of the mass distribution, and consideration of factors affecting the accurate representation of vibrational characteriatics of the model. Other resources required for performing efficient model checkout (2), and techniques used for different types of vibration analyses, were also developed $(3,4)$. The portion of this effort which is related to the dynamic aspect of these studies is the subject of this paper.

In the first section of this paper, the procedures used in converting the static model into a dynamic model are discussed. These include steps such as addition of non-structural components and extra grid points for the representation of the locations of the heavy mass items and inclusion of additional effects in shear panels to make them properly effective. For the accurate representation of the mass distribution, which is a key factor in a dynamic analysis, a procedure is developed which is discussed next (6). This procedure is used to systematically distribute the mass data over entire finite element model. In addition to these changes, a set of model quality assurance procedures is developed which helps the analyst to rapidly identify modeling errors prior to performing any analysis. These procedures are summarized.

In the second part, the efforts spent on obtaining an experimental database deacribing the vibrational characteristics of the aircraft structure and the finite element model enhancenent process are discussed ${ }^{(6)}$. The procedure used for testing, the criteria used for mode identification, and a summary of test results is discussed. A summary of correlation studies performed to check the validity of a finite element model and the associated modeling assumptions will be discussed. As a part of this enhancement process, the modeling problems that were identified as a result of correlation studies are presented. In addition, the general application of the test results is discussed.

Finally, use of a model reduction procedure for the 
purpose of increasing the computational efficiency for the dynamice optimisation applications is discused. A preliminary atudy wa performed to demonetrate application of auch a procedure. The lessons learned from this study which include the development and application of the model reduction process and the use of the reduced model in a sensitivity analysis study are summarized. Finally the lessons learned from the overall DAMVIBS effort are discussed.

\section{Vibration Modeling}

Generally, there are two major tasks involved in the development of a dynamic model from a static model. First step involves the additional modeling resources necenaary to make a static model useful for vibration analysis. Next is the generation and addition of the mass distribution to the static model.

A static model which is primarily used for calculations of internal loads and stresses under static loads generally does not contain a detailed representation of mase information. Prior to its use for dynamic analysis, it is required that additional steps be taken to accommodate for the locations of heavy mass items such as engines, misailes, etc. In the case of Apache nodel, shown in Fig. 1, one of these steps involved the addition of secondary structures and non-structural parts and a set of additional grid locations required for proper representation of masses. These grid points wore properly linked to the

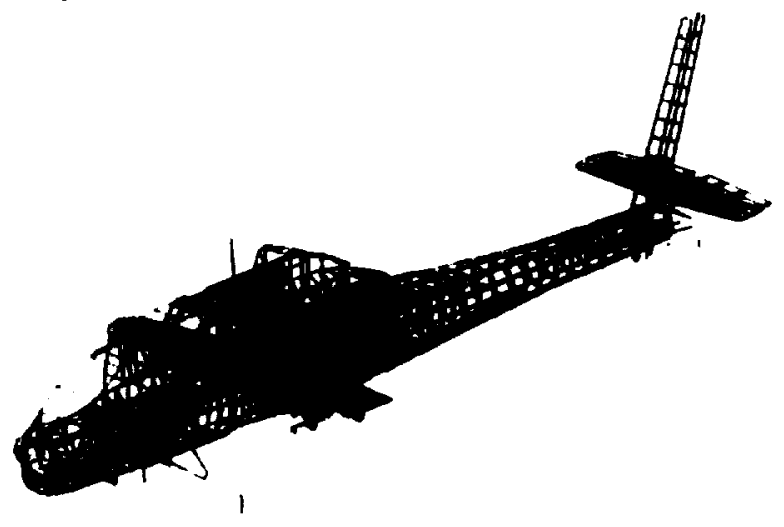

Fig. 1. AH-64A Dynamic MSC/NASTRAN Model.

surrounding areas. The next step in this conversion process was to correctly represent the structure for the in service conditions. In the case of static models, generally impending failure conditions are considered. In this case it is assumed that the skine are fully buckled and do not carry any tension/compression loads. This asoumption needs to be modified for simulation of in service conditions. Thua, proper steps were taken in order to make sure that the okins were appropriately effective.
Another task in generating a dynamic model involves the development of the mass model. This process generally conoiste of following tasks; a) generation of a detailed weights record for the weight empty tight confguration, b) generation of a detailed listing of useful load weights, c.g.'s, and inertias, c) diatribution of primary structure weight via material density parameter, d) manual distribution of large concentrated mass items (i.e., main rotor, transmission, engines, etc.), and e) automatic distribution of remaining mass data using a systematic and preferrably automated mass lumping process.

Most of the information specified in the above list is generally available through the mass database and the mass density information of different geometric representation of various model components. For the purpose of distributing the the remaining mass data (i.e., item e), an automated mass lumping procedure was established. In the case of Apache model, this procedure was used to systematically distribute the mass data over the opecified dynamic model grid locations and as a result a set of CONM2 cards were generated that were used in the dynamic model. The methodology used for the distribution of the finite element model mass data is discussed in detail in Ref. 5.

For the distribution of mass data, the lumping program requires two sets of information, namely, the actual aircraft mass data records and the location of the selected grid points of the finite element model. The mase data is organised and stored in a format that is consistent with the MIL-STD1374A. Starting with the frat maso item in the database, the program internally generates an imaginary volume, referred to as lumping volume, around the mass item. Next, by searching through the specified grid points, it identifies those grids which are confined within this volume and then assigns a different portion of the mass item to each of the selected grid points. Then, in case where there are still some remaining portions, the program increases the sise of the lumping volume and starts asoigning portions of the remaining mass item to the new set of grids which may now be within the confinement of the increased volume. This process is repeated until the mass item is completely distributed to the surrounding grids. This process is repeated for each of the mass items. However, proper care is taken within the program for avoiding the relumping of the mase of those items which have already been accounted for.

Figure 2 represents the general arrangement of the lumping process and illustrates the input and output streams of the automated mase lumping program. Although this program can accommodate structural mass input, the primary intent in the development of the program was to distribute nonstructural mass 
itens (e.g. fasteners, wire bundles, etc.) to model grid point locations. This mass lumping process minimises the human error through automatic generation of a set of CONM2 cards which are readily used as

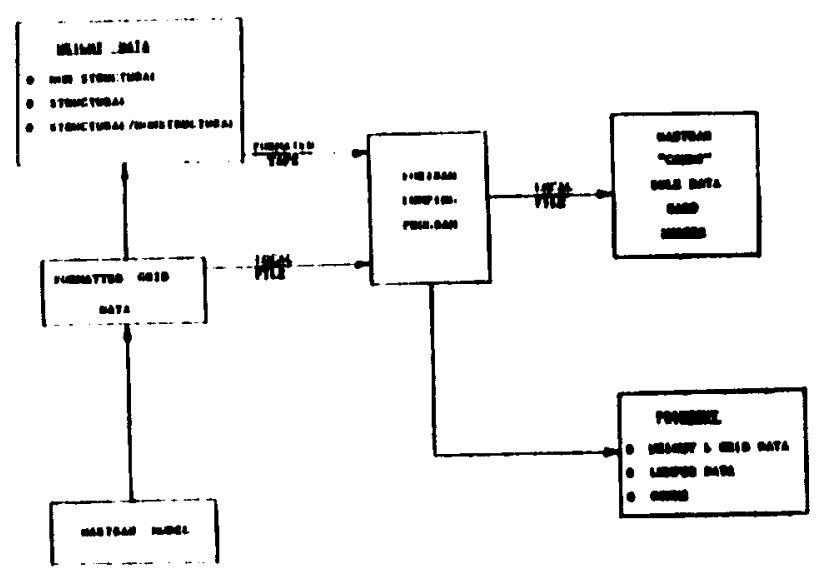

Fig. 2. Block Diagram of Mass Lumping Program.

a part of the NASTRAN bulk data deck. The atructural mass (e.g. skins and stringers) is calculated via material density cards interually within NASTRAN to form the total mass matrix.

\section{Model Verification Process}

Model verification process is one of many tasks requiring a substantial amount of time. Thus, it is very deoirable to have tools that can facilitate and automate this process as much as possible. A series of computational procedures were developed in the form of DMAPs. These DMAPs were used to identify both modeling errors and also provide certain information regarding identification of modes, energy associated with each mode and calculation of modal participation factors. The latter two DMAPa were developed prior to start of DAMVIBS program at the MDHC. However, due to their high level of effectiveness and also for the sake of completeness they are included here and are briefly discussed in the following sections.

As a result of applications of these modeling checkout DMAPs to a variety of structures, a common set of sources of errors has been identified. Those errors which occur during the assembly of the G-set stiffness matrix are due to; $(a)$ the improper apecification of the coordinate couplings where two components which are located in different coordinate syatems are coupled together improperly, (b) the use of a short beam element next to a long beam element, (c) use of a large or improper aspect ratio for plate elements, or (d) the use of CELAS elements between noncoincident points. Each of these modeling practices will result in the ill-conditioning of the G-set stifness matrix. In the second and third levels of model forma- tion, where the $\mathrm{N}$-set and F-set stiffness matrices are being assembled, improper applications of the MultiPoint Constraint equations (MPCs) and Single Point Constraints (SPCs) could result in further modeling errors. Incorrect specifications of these types of conatraints will result in incorrect representation of the linear relationships among different DOF, and overconstrained boundary conditions, respectively.

\section{Cholesky Decomposition DMAP}

The purpose of this check is to identify singularities or mechanisns as well as near singularities or soft spots in the model at the F-set level. For this purpose, a DMAP was developed which employs the DCOMP functional module to perform a triangular decomposition of the F-set stiffness matrix in the static solution sequence ${ }^{(2)}$. After the decomposition, a diagonal matrix results whose elements provide information about the conditioning of the stiffness matrix. A max factor diagonal value (i.e., ratio of the largest diagonal term to the lowest diagonal term) of over $10^{6}$ indicates a soft spot (i.e., near singularity) while a value over $10^{10}$ is indicative of a mechanism (i.e., singularity). In addition to the $\max$ factor ratio, another parameter, $\epsilon$, is calculated. Aside from round-off errors, any large nonzero value of $\epsilon$ is indicative of a singularity in the stiffness matrix.

\section{Multi-Level Strain Energy DMAP}

In contrast to the Choleski Decomposition Check, Multi-Level Strain Energy Check provides information about modeling problems at all three levels (i.e., G-set, N-set, and F-set) of model formation in NASTRAN. Through a DMAP, the stiffness matrix, and the rigid body modes obtained from the grid point geometry of the structure, are used to calculate the strain energy of the structure at each of the three levels of model formation. The same information is also used to calculate another parameter referred to as the nodal strain to provide further information about modeling probleme at each of the levele (2). As a result, any problems which might be caused by the improper grounding of the structure (i.e., G-set), incorrect application of MPC equations (i.e., N-set), or incorrect definition of the SPC constraints (i.e., F-set) will be identified through examination of these nodal strain energy at each level. To demonatrate the applicability of this powerful tool, it was applied to the AH-1G NASTRAN model (2). Examination of the Nset strain energy matrix, shown in Tablo 1 , revealed some problems associated with the rotational degrees of freedoms (i.e., the third through sixth diagonal elements of the matrix are much larger than 10) which are indicative of incorrect application of MPC equations. To further identify the degree of freedom associated with the MPC equation, another matrix, re- 
ferred to as N-set nodal strain matrix was examined. The last

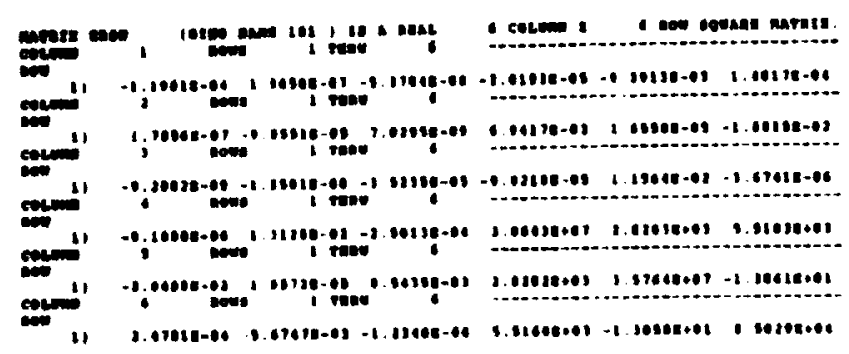

Table 1. N-set Strain Energy Matrix.

three columns of this matrix, shown in Table 2 , which were representative of the rotational degrees of freedom revealed the location of the troublesome grid and the associated degrees of freedom (i.e., grid points 7505 and 15218). Once the asoociated MPC equation was corrected the DMAP was again applied and aimilar problem associated with the MPC equation used for the engine support location wa identified. This problem was then corrected.

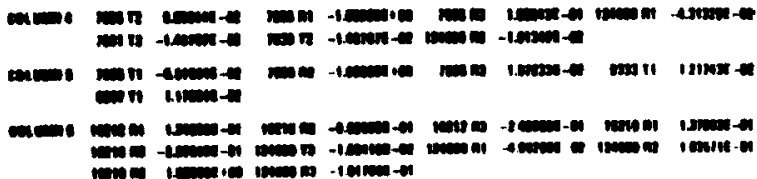

Table 2. N-set Nodal Strain Matrix.

\section{Kinetic Energy DMAP}

One of the key recent improvements in the dynamic analyois of large finite element models is the development of efficient and accurate eigenvalue extraction techniques. Introduction of such techniques has rosulted in their application to more complex syatems and conequently much larger FEMs. As a result, for the prediction of atructural modal characteriatics, the analyst is often confronted with the identification and post processing of large number of high density modal vectors. For very large and detailed models, this proceas is rather tedious and time consuming. Consequently a DMAP wa developed which is referted to as Kinetic Energy Check DMAP (3.4). Application of this DMAP results in the caculation of the modal kinetic energy associated with each mode and rapidly identifies the candidate structural modes. Additionally, auch results provide the necessary information to ideatify the local modes and any poseible modeling errors. Since the development of this DMAP, it has been applied to a large number of different structures and has reaulted in a subatantial saving of manhoure and the elimination of costly and time consuming plotting process of the local/trivial modes.
This technique was initially applied during dynamic analysis of the Hughes Advanced Attack Helicopter (AAH). At the time of application, the model had 9792 degrees of freedom. To identify the modal characteriatics of the model, an eigen analysis was performed up to $25 \mathrm{~Hz}$. Within this frequency range, eighty seven modes were calculted where each mode contained an eigenvector containing 10000 values. Through application of the kinetic energy DMAP, a filtered modal kinetic energy matrix was obtained which contained only a set of larger values for each mode (1). A quick examination of the filtered modal kinetic energy matrix revealed candidates for the local or actual structural modes. A structural mode is generally identified by a column containing many large numbers, since the energy is distributed and large portion of structure moves together. In the case of local modes, only a few elements of the matrix have very large values, indicating only a local area of structure is moving. Figure 3 shows a sample of a structural mode identified through this procedure.

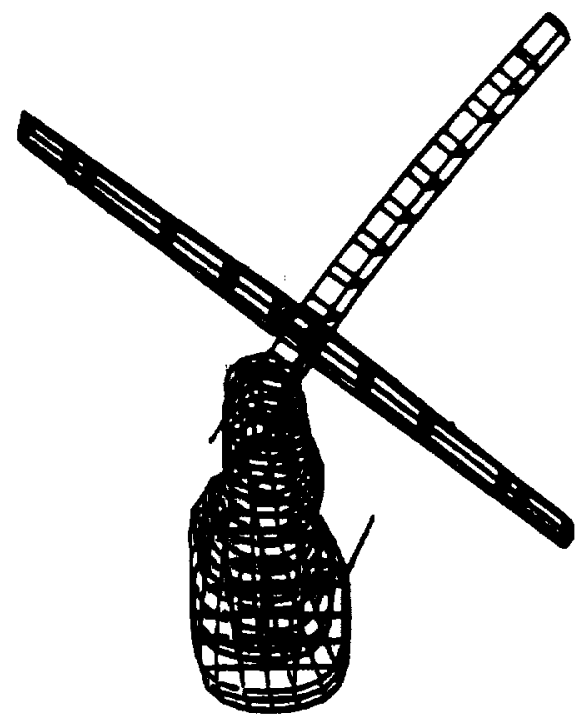

Fig. 3. AAH Firat Torsion Mode.

Modal Participation DMAP

An important information during studies of the dynamic structural response of linear systems is the contribution of different structural modes to the total response of a selected point or a finite region of the structure. Such information generally providea valuable insight into the vibrationel characteristic of the system and points out areas where structural modifcations could result in improved response. In certin applications, auch data provides the necessary information for the identification and selection of an optimum location for vibration control devices. For this purpose, a DMAP was developed which calculates the modal participation factors associated with response of a selected location of the structure ${ }^{(7)}$. For the ap- 
plication of this DMAP, frequency response (SOL 30) is used. Application of this technique to a vibration reduction study is discused in detail in Ref. 5.

\section{Experimental Efforts}

\section{Test Objective}

The main objective of the shake test was to obtain an experimental data base describing the vibration response of the test vebicle. This data base consisted mainly of transfer functions of response acceleration to input force versus input frequency for various measurement locations throughout the fuselage structure. Although the lest was not a modal survey test, the major airframe modes were extracted from the test data. These modes provided ineight into the basic vibration characteristics of the fuselage and aided in the correlation with NASTRAN model.

\section{Tent Vehicle}

The teat vehicle used was the U.S. Army's AH-64A Advanced Attack Helicopter. The teating was performed with the vehicle in the primary misoion conGguration (0). The main rotor hub and blades were replaced with a rigid steel fixture which was attached to the static mast in a manner similar to the actual hub. The dummy structure was equivalent to $100 \%$ of the weight of all of the actual items except for the rotor blades. The blade weight was reduced to $60 \%$ of the actual weight to more closely represent the dynamic equivalent of the rotating system.

\section{Teat Setup}

The teat vehicle was suspended from a gantry. Air suspenaion oprings located at the top of the gantry provided vibration isolation between the test vehicle and the gantry structure. This method minimised feedback from the gantry modes of vibration into the teat aircraft. Link chain was used to attach the air spring system to the dummy main rotor hub. This technique removed the tendency for the aircraft to yaw. Sinusoidal vibration excitation was applied to the test vehicle at the inain rotor hub and at the tail rotor. Three forces (longitudinal, lateral and vertical) were applied to the main rotor hub, as well as two moments (pitch and roll). These forces and moments were applied at the vertical and atation positions equivalent to the blade plane and the center of rotation. Three forces were applied at the tail rotor location: longitudinal, lateral and vertical. The tail rotor excitation point was at the center of rotation and at a lateral position which is half way between the two rotor planes. The flight tail rotor equipment was on the aircraft during the entire shake teat.

\section{Test Procedure}

During excitation, data was taken over a frequency range of 3.75 to $50 \mathrm{~Hz}$ except for the tail rotor input cases which extended this range to $200 \mathrm{~Hz}$. The frequency was stepped up in increments of $0.5 \%$ and the input dwelled for a few seconds at each frequency step to allow the response to come to quasi-steady state. Data was taken for all accelerometers at each frequency step. Each dwell and measurement step took about 5 seconds to complete, so an entire test $(3.75$ to $50 \mathrm{~Hz}$ ) could be run in less than 45 minutes.

\section{Instrumentation}

A total of 102 accelerometers were mounted on the aircraft at 39 locations and four were mounted on the gantry and suapension system. Each accelerometer was lested in a mechanical system, comprised of a load cell and a known free mass. The mass was driven in the test frequency range. The resulting transfer function from each unit was used by the computer to convert voltage to acceleration and to adjust the phase at each frequency step.

The accelerometer locations were chosen based on three criteria: (1) to identify the response at key locations such as pilot seats, engines, hubs, etc.; (2) to describe fundamental modes of the airframe; and (3) to identify important local modes such as those of the wings.

The accelerometers were mounted on one inch cubic fiberglass blocks which were epoxied to the airframe. The blocks were oriented such that their faces were aligned with three orthogonal axes of the ship. Each block had one to three accelerometers attached to it.

The use of a large number of fixed accelerometers proved to be much more effective than a few roving accelerometers, as is usually done in this type of testing. The chief reasons for this are as follows. The accuracy of accelerometer positioning was greatly increased. Errors in mounting and polarity were minimized because the accelerometers remained fixed in position for the entire test. Moreover, the time required for testing was greatly reduced because only one frequency sweep was necessary for each test case.

\section{Data Acquisition and Control}

The system used for data acquisition and control was $100 \%$ computer controlled and exclusively used Hewlett Packard instrumentation. At the heart of the system was the HP9836 computer/oystem controller.

At each frequency step, all data channels were scanned and 16 readings were taken over one cycle of vibration. This data was then sent from the HP6942A scanner 
to the HP9836 computer where magnitude and plaase data wa extracted by mean of a Diecrete Fourier Tranoform. By this method the firat harmonic was extracted while the higher harmonics, up to the 8th, were rejecled.

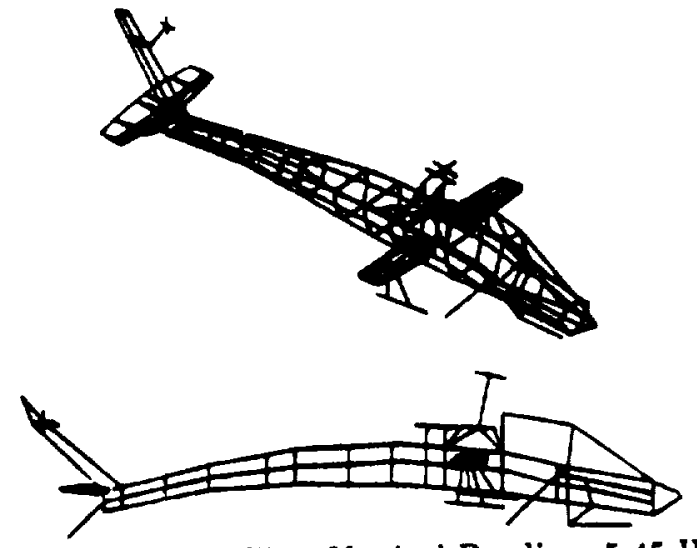

Fig. 4. Fuselage First Vertical Bending, $5.45 \mathrm{~Hz}$.

\section{Test Reaulte}

The essential results of the shake test were the response tranofer functions. Additional results include natural frequencies and mode shapes which wore gleaned from the transfer functions. Although not essential, this data aided in the correlation of the NASTRAN model and provided insight into the vibration characteriatics of the aircraft. A sample mode shape from the teat data is presented in Fig. 4. Natural frequenies were eatimated from the data by means of an indicator function ${ }^{(0)}$. When the value of the firat function approaches unity, a matural frequency is indicated. Sample indicator function results are shown in Fig. 5.

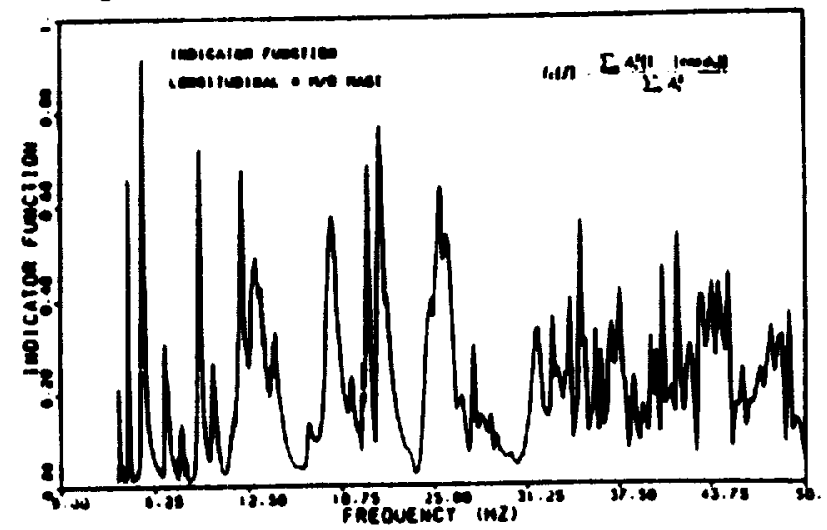

Fig. 5. Sample Indicator Function Reoults.

\section{Correlation and Model Enhancement}

An excellent correspondence between test and NASTRAN reeults was obtained for the frequency responees up to $13 \mathrm{Hs}$. This includes the first six modes found in the shake test. Above $13 \mathrm{Hs}$, the correla- tion tends to deteriorate gradually as the frequency of the response increases. A comparison of natural frequencies found by test and calculated by NASTRAN is given in Table 3 . Typical response correlation plote are shown in Fig. 6.

\section{DOSORLPTIOY}

TAILOCM TORSION

LST VERTICAL BONDDN

1st LATERAL BONDDX

LONC. BandDE OF VERIICAL MR MAST BONDDG, LONC. MR MAST BONDDE, LAT. SAREIRIC WOAC BDNODN ANTI-SYMA. WDE EDDDC STABILATOR YN

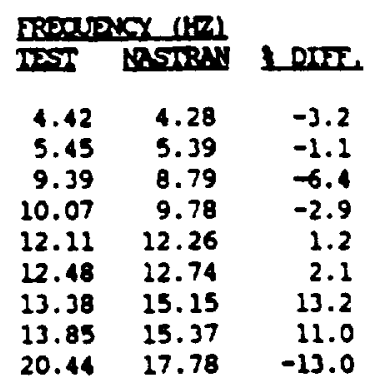

Table 3. Correlated Modes

The problem areas in the correlation were the engines, stabilator, and the wings. However, much inprovement was obtained in the vertical response of the wings based on the preliminary correlation results. In addition to improving the existing finite model, much knowledge gained in the correlation effort will be applicable to future analyses. Shake test results can often be used to refine an existing finite element model. This is accomplished by comparisons of experimental and analytical results. This comparison initially indicates whether or not there are errors in the model and acoints in locating probable areas in which these errors may reside. If an error has been caused by an incorrect asoumption, then the correlation study can provide a guide as to how the problem might be corrected. Finally, the teat comparison provides a standard by which the degree of enhancement can be measured. The following discussion describes an example of how shake test results were utilised to enhance the NASTRAN finite element model of the AH-64A airframe.

Initial comparison of the frequency response curves obtained from the test with those predicted by NASTRAN indicated that, for some cases, there were discropancies between the two sets of results. The most noticeable problems appeared in the responses of the wings, the engines and the stabliator.

Examination of the deflected shapes obtained from teat and analyois helped to further localise and identify errors in the model. A specific example of such error is a mode at approximately $8.5 \mathrm{Hs}$ which is shown in Fig. 7. This mode is predominantly a wing mode although it is significantly lower in frequency than the wing modes found in the test results. In addition, one wing undergoing pure vertical bending, while the other is experiencing nearly pure wing torsion. Such asymmetric behavior was not found in the test results. Therefore, it was concluded that there were errors in the wing portion of the Gnite element model. 
After clowe acrutiny of the wing portion of the model, problems were identified and corrected. The problems were mainly aymmetries in the lumped masses and element products of inertia, overly stiff modeling of the pylons, and the omiacion of the trailing edge. The wing trailing edge was ignored in the static model because it was considered secondary structure.

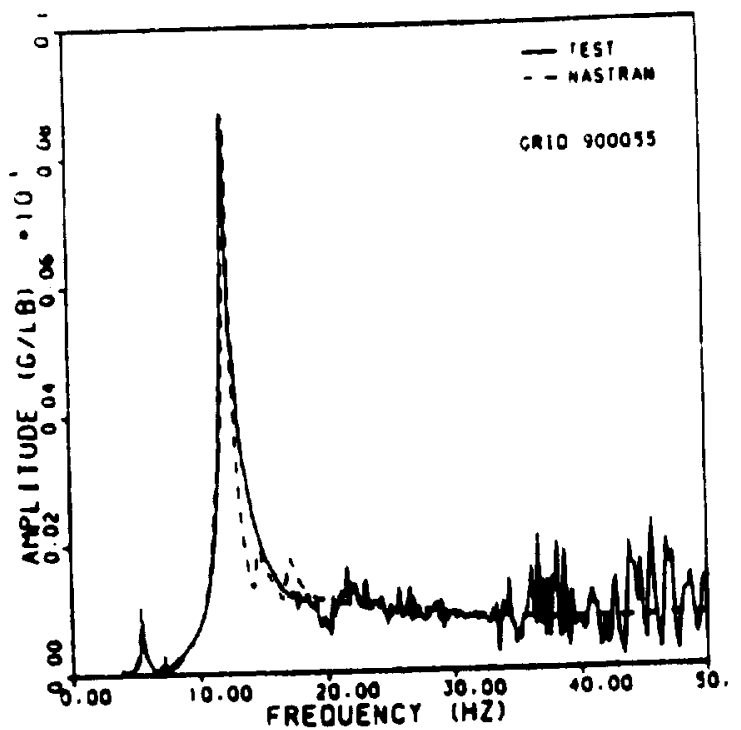

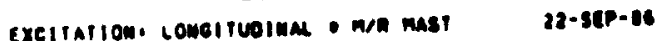
valu nolon we. Lomalivolume aespomse

11.241 .5
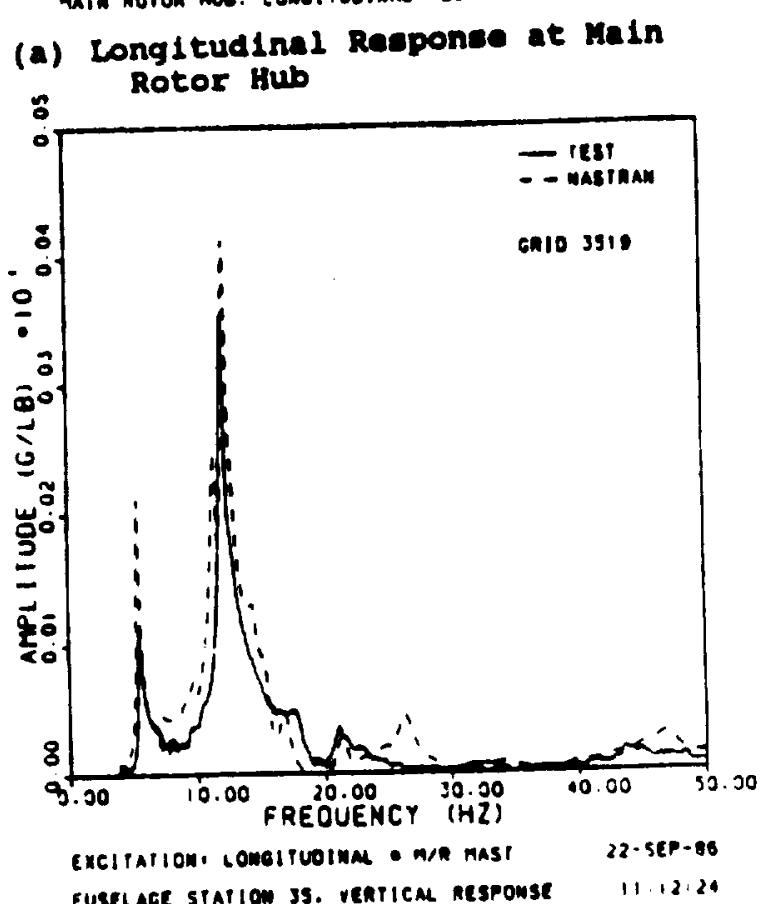

(b) Vertical Response at Aircraft Nose
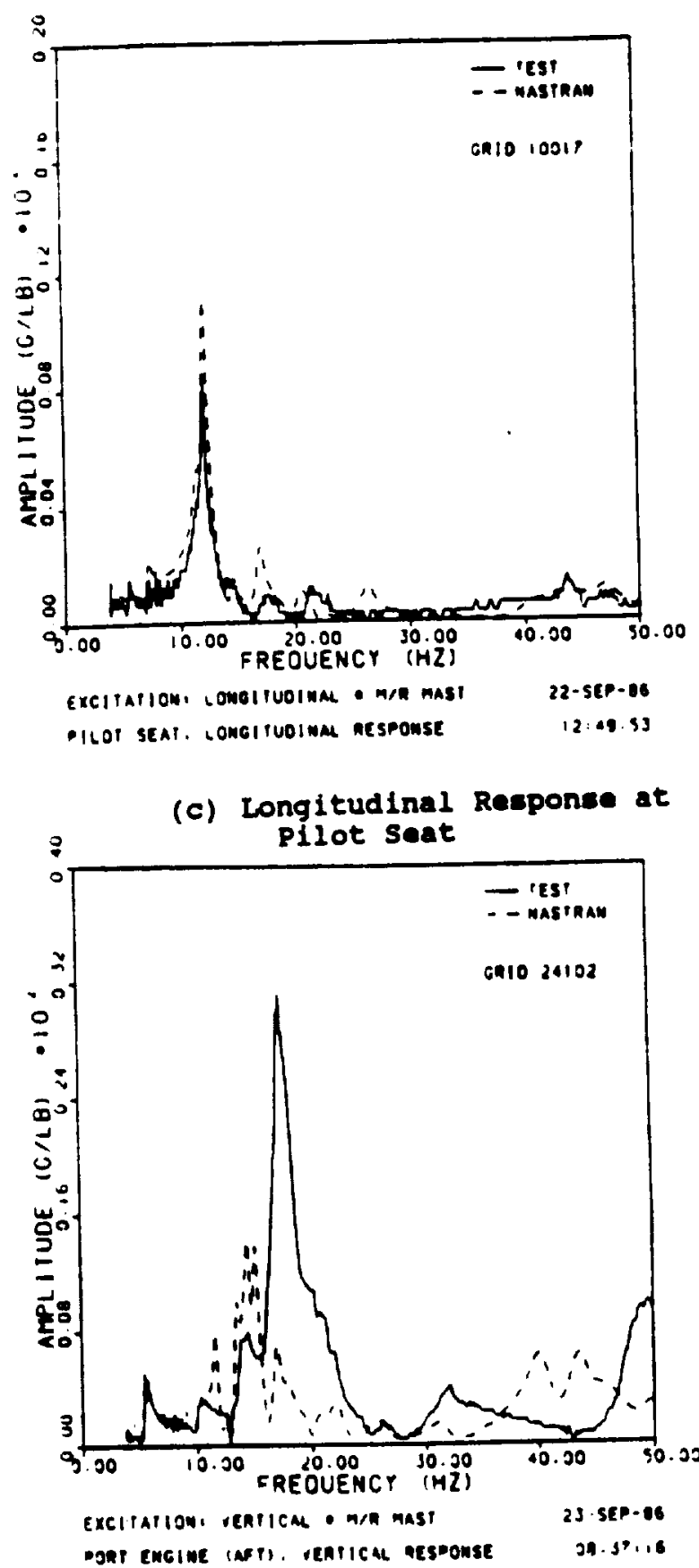

(d) Vertical Response at engine

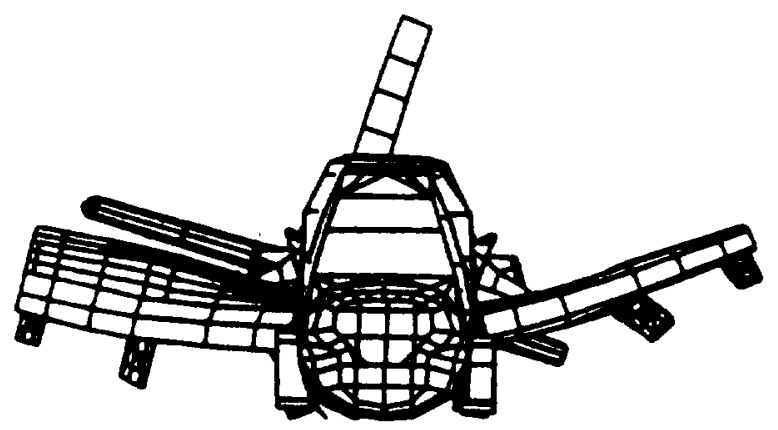

Fig. 7. Wing Mode at $8.5 \mathrm{Hs}$. 
With the improvements made to the model, the analyst can use the entire inventory of test results such as frequency response plots, deflected shapes, and modal frequencies to examine the effect of the improvements. An excellent example of this is the improvement of the vertical reoponse of the wing as a result of the changes implemented in the model. Soe Fig. 8.

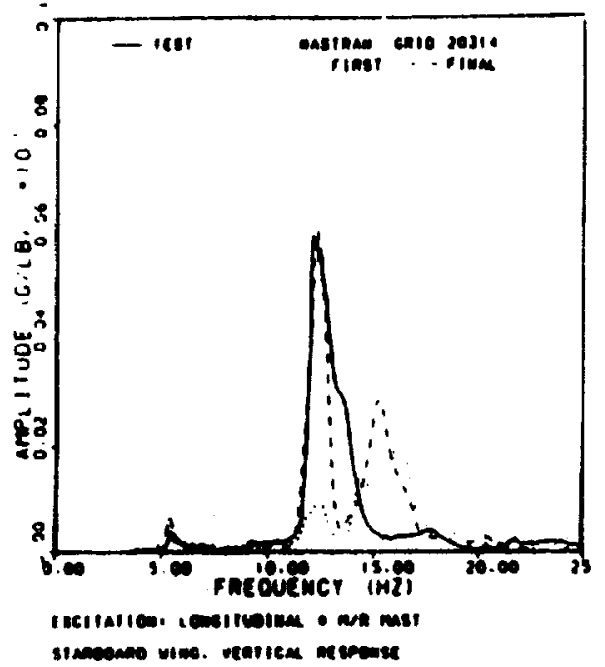

Fig. 8. Improved Correlation of Vertical Renponee at Wing Tip.

\section{Vl. General Application of Test Results}

A comprehensive shake test, such as the one performed under this program, not only helps to enhance an exieting finite element model of the teat specimen, but also provides valuable guides for enhancing other models and future modeling efforts. Teat results may be used to verify modeling practices uned in the past, to identify questionable asoumptions and practices, and to provide a reasonable lovel of confidence in the analysis.

It is usually neceasary to make acoumptions in proparing a finite element model. The validity of these assumptions must be verified by testing. In this particular case, the teat results confirmed many of the asoumptions used in modeling the AH-64A helicopter. For example, the asumption that stringers and longerons carry axial loads while skins and webs carry shear loads was verified. The effectiveness of using a static model for dynamic analysis with dynamic reduction was also confirmed. Good correlation associated with main rotor mast responses confirmed our modeling tochnique regarding the main rotor support structure and the overall representation of the airframe.

The correlation of the test results revealed some as sumptions and practices which were not quite correct. The following are some examples. Although using a static model for dynamic analysis was generally successful, some of the assumptions made for statics were not good for a dynamic model. Two examples were ignoring the trailing edge structure of the wing and the frictional load carrying capability of bolts. Some assumptions made for statics were previously recognised and corrected to meet dynamics requirements. An example of this was the shear panel effectivity. Regarding the mase distribution, some of the assumptions and methode were not strictly correct. For example, the automated mass lumping routine does consider connectivity when lumping the masses, nor does it rigorously account for rotational mass moments of inertia.

\section{VIL. Vibration Reduction Studies}

Generally, use of model reduction procedures for the purpose of increasing the computational efficiency is of great interest in a dynamic analyeis. This is especially true in dynamic optimisation applications. Consequently, a preliminary study was done to explore the applicability of a reduction procedure to a vibration reduction study. A model reduction procedure was initially developed and was used in vibration study.

\section{Model Reduction Procedure}

A computational procedure for the reduction of large airframe finite element models was developed (8). This procedure, which has been implemented as a set of DMAPs, results in a significantly reduced model while retaining the essential dynamic characteristics of the full-sised model. This procedure was applied to a slightly modified vervion of the airframe dynamic 6nite element model of AH-64A Attack Helicopter. A mathematicaly reduced model with significantly less DOFs was obtained. This reduced model, is shown in Fig. 9.

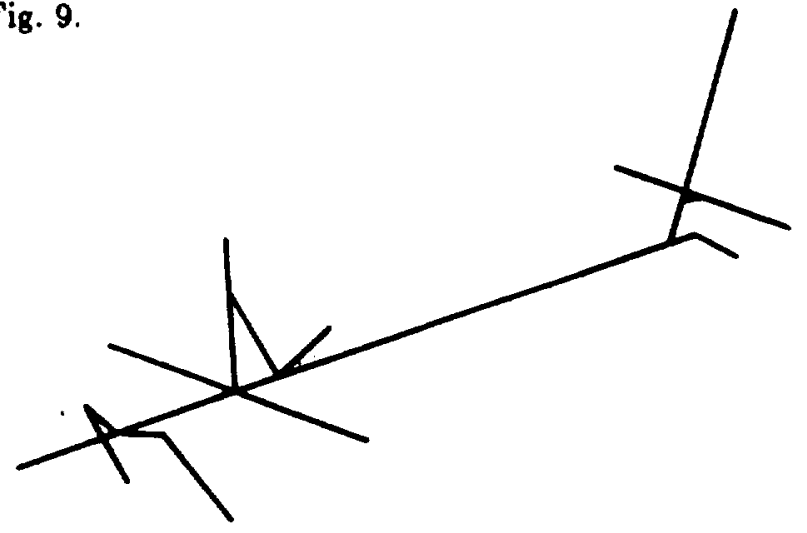

Fig. 9. Apache Stick Model.

This model is an accurate representation of the global behavior of the full model. The resulting reduced model resulted in a substantial reduction in the computation time. This reduced model which has 83 grid 
points and a total of 498 DOF is only a mathematical representation of the reduced (stick) model (i.e., the elastic properties are defined in terms of stiffness matrix rather than physical elements such as bar, beam, etc.). However, further steps could be taken to carry this mathematical reduced model one step further and develop an equivalent "physical" reduced model. This process was not pursued. Instead, the "mathematical" reduced model was adapted for sensitivity analysis study which will be discussed in the following section. Following the reduction process, an eigen analysis was performed to evaluae the accuracy of the dynamic reduced model. Figure 10, represents the first vertical bending mode.

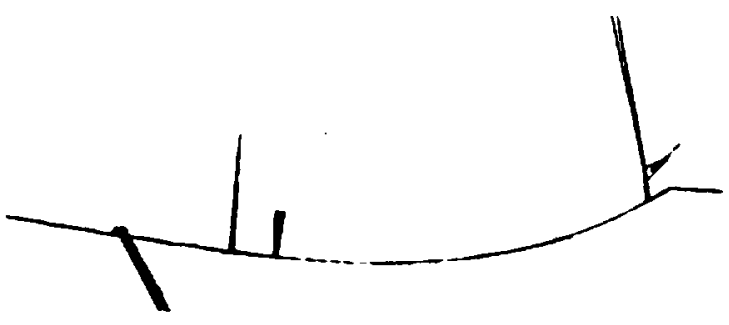

Fig. 10. Stick Model First Vertical Bending.

To further quantify the degree of correlation between the two models, an in-house computer program which provided a systematic way of checking the degree of correlation between frequencies, mode shapes or a combination of the two, was used (8). Table 4 shows the resulting correlation matrix between the two models.

\begin{tabular}{|c|c|c|c|}
\hline \multirow[b]{2}{*}{ MODE } & \multicolumn{2}{|c|}{ FREQUENCY (HZ) } & \multirow{2}{*}{$\begin{array}{l}\text { MODE SHAPE } \\
\text { CONRELATHON }\end{array}$} \\
\hline & FULL & STICK & \\
\hline TATLBOOM TORTSION & $\overline{3} . \overline{45}$ & 5.6 & $6 \%$ \\
\hline IST VERT BENDING & 600 & 6.15 & 0.93 \\
\hline IST LATERAL BENDING & 1070 & 9.76 & 084 \\
\hline SYM ENGING YAW AND PITCH & 1114 & 1167 & $0 \infty 0$ \\
\hline VERT TALL WONG AENDING & 1197 & 12.31 & 091 \\
\hline MAST IONG. BFNIIING & 13.1 & 11.33 & 087 \\
\hline ANTISYM ENGINE YAW & 1416 & 16.43 & 070 \\
\hline STABILATOR YAW & 2063 & 19.60 & 081 \\
\hline
\end{tabular}

Table 4. Modal Correlation Matrix.

The correlation factors indicated in Table 4 are basically indicative of the degree of correlation between a pair of global modes.

Vibration Reduction study

In addition to the development and application of the model reduction procedure, another study was made to examine the applicability of the reduced (stick) model to a vibration reduction study. For this purpose, the reduced model was subjected to different four-per-rev hub excitations and the modal frequency responses (SOL 30) of different locations of the structure, together with the contribution of each mode to the total response of each location, were calculated (7). Subsequent to the identification of the dominant modes, a design sensitivity analysis study (SOL 53) was performed to identify the pertinent model parameters (e.g., cross sectional area, area moment of inertia, etc.) which have the most effect on the vibrational response at the selected locations. Once these parameters were identified, certain incremental changes were made to each of them individually or a combination of these parameters to reduce the vibration at the selected locations.

In conjunction with the vibration reduction study, the contribution of each mode to the total response of the selected portion of the structure was determined at the location of interest. This location was the tip of the vertical tail of the AH-64A reduced model. For this purpose, an in-house DMAP (7) program was used in conjunction with the MSC/NASTRAN modal frequency response solution sequence (SOL 30). Figure 11 shows the four modes with the largest contributions to the total response of the selected point, when the aircraft is subjected to a four-per-rev vertical hub excitation.

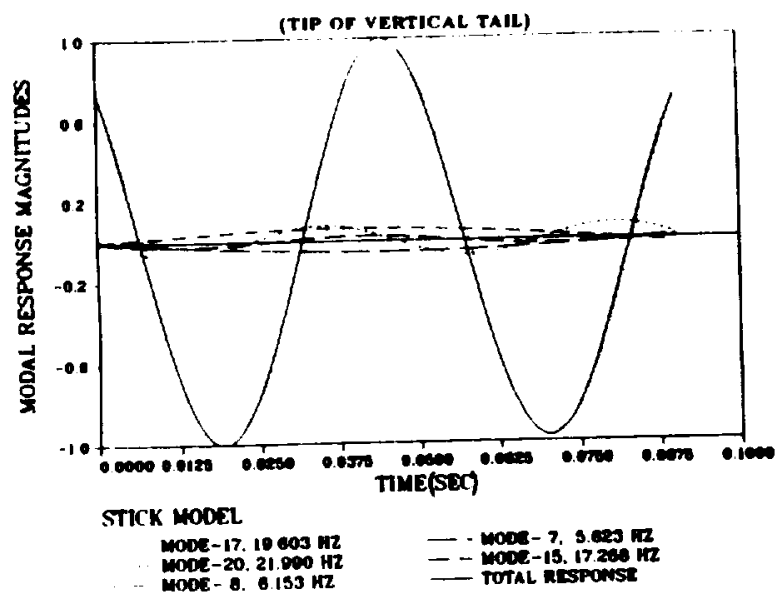

Fig. 11. Structural Modal Response.

As can be seen, mode 17 contributes the most to the total response. In addition, examination of other modes and the results obtained from the DMAP indicated that the fifteenth mode (i.e., $17.268 \mathrm{~Hz}$ ) to be the next highest contributor to the response of the point of interest. Therefore, these two modes (i.e., modes 15 and 17) were selected to be used in a following design sensitivity analysis. This selection process was repeated for two other types of hub excitations (i.e., longitudinal and lateral excitations) and a similar pattern was obtained.

To identify those parameters which have the most effect on the response of selected normal modes in the area of interest of the structure, a sensitivity analysis was performed. For this study, the tailboom which 
is in the close proximity of the vertical tail was selected. The cross-sectional area, the two area moments of inertia, and the torsional constant parameters were selected as the design variables. The two dominant stick model modes (i.e., modes 15 and 17) which represented mainly the tailboom vertical and lateral bendings, were selected as the "constraint" parameters. The design sensitivity analysis was perforned for all twelve tailboom frame segements and a set of sensitivity coefficients obtained. These are shown in Fig. 11 and 12.

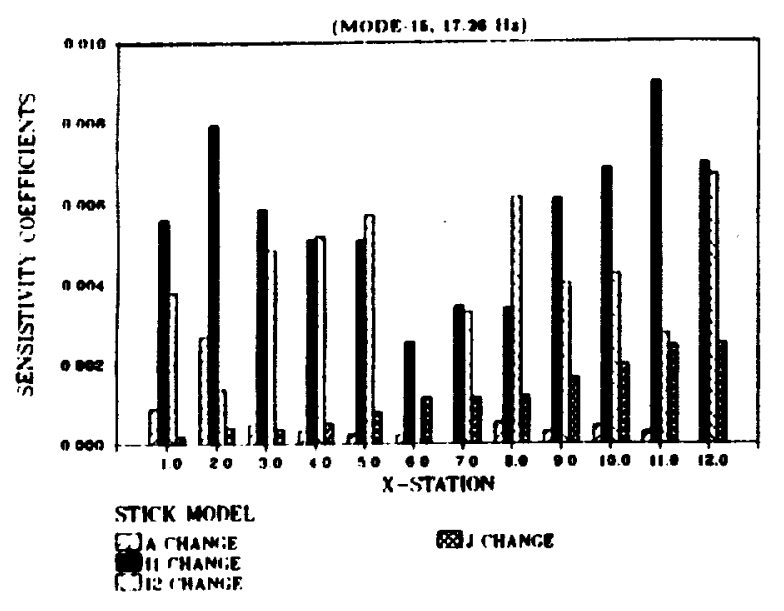

Fig. 11. Sensitivity Coefficients, Mode 15

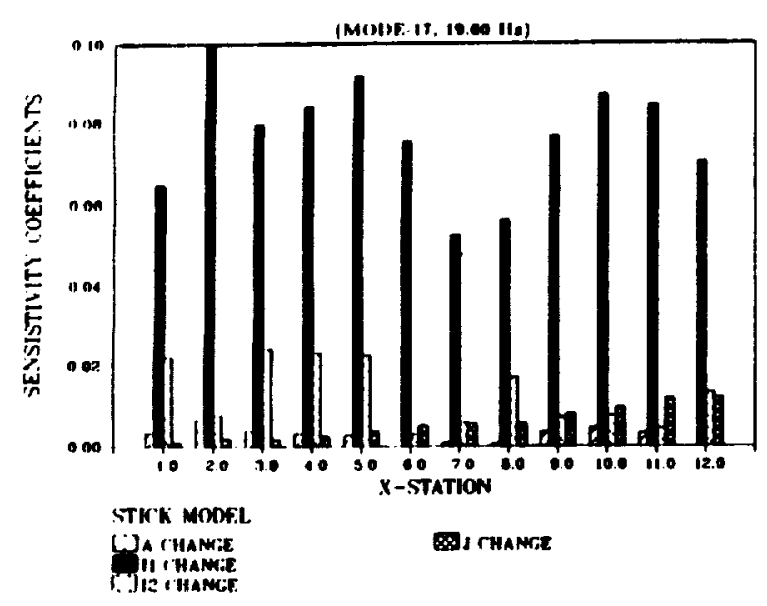

Fig. 12. Sensitivity Coefficients, Mode 17

Examination of these figures revealed that the two area moments of inertia parameters have the most effect on the frequency placement of the two selected modes. Consequently, these parameters were altered which shifted the frequencies of these two modes away from the four-per-rev excitation frequency ${ }^{(9)}$. This process resulted in a reduction of the vibration level at the tip of vertical tail. Similar changes were also made to the same locations of the full model and the response of the vertical tail was calculated.

\section{Concluding Remarks}

A summary of the MDHC's effort related to the development of DAMVIBS dynamic modeling guidelines, testing and the correlation studies has been presented. As a result of this work, a set of comprehensive modeling procedure and check out tools have been generated which have been used in modeling of large airframe structures. These procedures which been used in the ongoing enhancement process of the exiting MDHC product models, have also been applied to the newly developed product models. Throughout this program, a substantial amount of experienced have been gained in creating other reliable airframe finite element models ${ }^{(10)}$ which are used during the design process. Through such improved modeling capability a better estimation for man-hour requirements and scheduling process is now possible.

In relation to performing shake tests and efficient use of this data, our capability has been substantially enhanced. This has been accomplished through use of experience gained during this program in the preperation of better test plans, performing the the shake tests where issues such as support of test structure, applications of loads or load levels are of great importance. Reduction and application of test data to the model resulted in improved corrolation methodology which also brought into focus the shortcomings associated with different correlation methods and the inaccuracy associated with higher analytical modes. Further examination of the test data pointed out the degree of structural nonlinearity and provided insight into damping characteristics of the aircraft structure.

The exchange of information between $\mathrm{MDHC}$ and other three companies provided a means to compare methodologies used by each group and the associated results. Such an exchange of information resulted in improving certain areas of our models. One of these areas was inclusion of secondary structural components which have resulted in improving the correlation results. Finall, these studies have pointed out new challenges in terms of limitations associated with FEA which need to be further investigated.

A summary of the lessons learned during these studies are listed as follow:

- The static finite element model may be used for dynamic analysis after making the proper modifications.

- The proper modeling of the mass distribution and representation of secondary and nonstructural components is essential to accurate vibration modeling. Automation of this procedure greatly reduces modeling time. 
- Model checkout and verification facilitates obtaining accurate results. Automated DMAP procedures enhance the analysts capabilities in this area.

- The performance of the finite element model can be significantly improved by the application of the shake test results.

- Utilization of stationary accelerometers reduces the test time.

- Prior to performing the test, magnitude and types of excitation loads should be studied using FEM.

- Excellent correlation with AH-64 shake test results was obtained at most measurement locations up to $13 \mathrm{~Hz}$.

- Knowledge gained in such a correlation study is also applicable to future analysis efforts.

- The vibration reduction obtained as a result of a preliminary dynamic optimization study demonstrated the applicability and benefits of the model reduction technique.

- Confidence in finite element analysis as a valid tool for predicting helicopter fuselage vibrations has been greatly increased.

\section{Acknowledgements}

The authors would like to acknowledge other individuals who contributed significantly to different phases of this program. These individuals are; Richard A. Christ, Friedrich K. Straub, Douglas A. Ferg, Kevin L. Kilroy, Grant R. Parker, John J. Brown, Leland F. Foote, Gregory J. Korkosz, Karan Sangha, and Joe Shamie.

\section{References}

[1] Christ, R., Ferg, D., Kilroy, K., Toossi, M., and Weisenburger, R., "Plan, Formulate and Discuss a NASTRAN Finite Element Model of the AH64A Helicopter Airframe," NASA Contractor Report 187446, October 1990.

(2) Hashemi-Kia, M., Kilroy, K., and Parker, G., "Development and Applications of a MultiLevel Strain Energy Method for Detecting Finite Element Modeling errors," NASA Contractor Report 187447, October 1990.

(3) Parker, G.R., and Brown, J.J., "Kinetic Energy DMAP for Mode Identification", Presented at MSC/NASTRAN User's Conference, March 1819, 1980, Pasadena, California.
[4] Kilroy, K., "Instruments That Overcom the Difficulties in Large Finite Element Analysis Prolems", Presented at the joint American Helicopter Society, Nanjing Aeronautical Institute Meeting on "The thoretical Basis of Helicopter Technology", November 1985, Nanjing, China.

[5] Ferg, D., "Automated Mass Distribution Program For Dynamic NASTRAN Modeling," Flight Technology Technical Note FTTN-89-005, McDonnell Douglas Helicopter Company, Mesa, Arizona, May 1989.

(6) Ferg, D., Foote, L., Korkosz, G., Straub, F., Toossi, M., and Weisenburger, R., "Plan, Execute, and Discuss Vibration Measurements and Correlations to Evaluate a NASTRAN Finite Element Model of the AH-64A Helicopter Airframe," NASA Contractor Report 181973, January 1990

(7) Parker, G.R., and J.J., Brown, "Evaluating Modal Contributors in a NASTRAN Frequency Response Analysis", MSC/NASTRAN User's Conference Proceedings, March 1983.

[8] Hashemi-Kia, M., and Toossi, M., "Development and Application of A Technique for Reducing Airframe Finite Element Models for Dynamics Analysis," NASA Contractor Report 187448, October 1990.

[9] Hashemi-Kia, M., and Toossi, M., "Finite Element Model Application to Parametric Studies and Optimization of Rotorcraft Structures," American Helicopter Society 46th Annual Forum, Washington D. C. May 1990.

(10) Ferg, D., and Toossi, M., "Finite Element Modeling of the Higher Harmonic Controlled OH-6A Helicopter Airframe," NASA Contractor Report 187449, October 1990.

(11) Sangha, K., and Shamie, J., " Correlation of AHIG Airframe Flight Vibration Data With a Coupled Rotor-fuselage Analysis," NASA Contractor Report 181974, August 1990. 
